\title{
Erfahrungen bei EXAM/ONYX-Klausuren
}

\author{
J. Hoffmann, A. Brosius \\ Professur Formgebende Fertigungsverfahren, Institut für Fertigungstechnik, Fakultät Maschinenwesen, \\ TU Dresden
}

\begin{abstract}
Ein Jahr digitale Lehre bedeutet auch ein Jahr digitale Prüfungen. In diesem Paper sollen dazu einige praktische Erfahrungen aus Sicht der Professur Formgebende Fertigungsverfahren (FF) zusammenfassend vorgestellt werden. Dabei ist der Einsatz der an der TU Dresden zur Verfügung stehenden Softwareplattform EXAM@TUD Schwerpunkt der Betrachtungen. Bei deren Nutzung gibt es neben vieler positiver Ansätze auch einige Schwachpunkte, die noch einer Verbesserung bedürfen. Diese sollen als Erfahrungsbericht aus ca. 60 Online-Klausuren (Probe- und Abschluss) hier näher vorgestellt werden.

One year of digital teaching also means one year of digital examinations. This paper will summarise some practical experiences from the perspective of the Chair of Shaping Manufacturing Processes (FF). The main focus is on the use of the EXAM@TUD software platform available at the TU Dresden. In addition to many positive approaches, there are also some weak points in its use that still require improvement. These are to be presented here in more detail as a report of experience from approx. 60 online examinations (trial and final).




\section{Vorbemerkungen}

Ein Jahr digitale Lehre bedeutet auch ein Jahr digitale Prüfungen. In diesem Paper sollen dazu einige praktische Erfahrungen aus Sicht der Professur Formgebende Fertigungsverfahren (FF) zusammenfassend vorgestellt werden.

Die Professur beschäftigt sich in der Lehre schwerpunktmäßig mit den Fachgebieten Urform- und Umformtechnik, Zerspan- und Abtragtechnik sowie Produktionsautomatisierung und Fertigungsplanung im Bereich der Teilefertigung. Entsprechend umfangreich ist das aktuelle Lehrangebot, welches seit dem Sommersemester 2020 wie an der gesamten TU weitgehend digital umgesetzt wird. Zur digitalen Lehre gehört auch das entsprechende Angebot an Prüfungen zum Nachweis des Lehr- und Lernerfolges.

Auf Grund des großen Lehrumfanges ergaben sich zum Abschluss des Sommersemesters 2020 insgesamt 16 sowie zum Ende des Wintersemester 2020/21 in Summe 41 Probe- und Abschlussklausuren, die alle digital in Eigenverantwortung oder in Kooperation mit anderen Lehrstühlen online realisiert wurden

(Abb. 1).

Die verschiedenen Klausuren wurden mit Hilfe des Systems EXAM@TUD realisiert (Abb. 2). Diese Software wurde von der Firma BPS Bildungsportal Sachsen GmbH speziell für die TU Dresden eingerichtet. Bei den Klausuren wa- ren zwischen 4 und 400 Teilnehmer zu verzeichnen. Der zeitliche Umfang der Klausuren betrug zwischen 80 und 180 min mit einem Anteil der Professur FF von 20 bis 90 min.

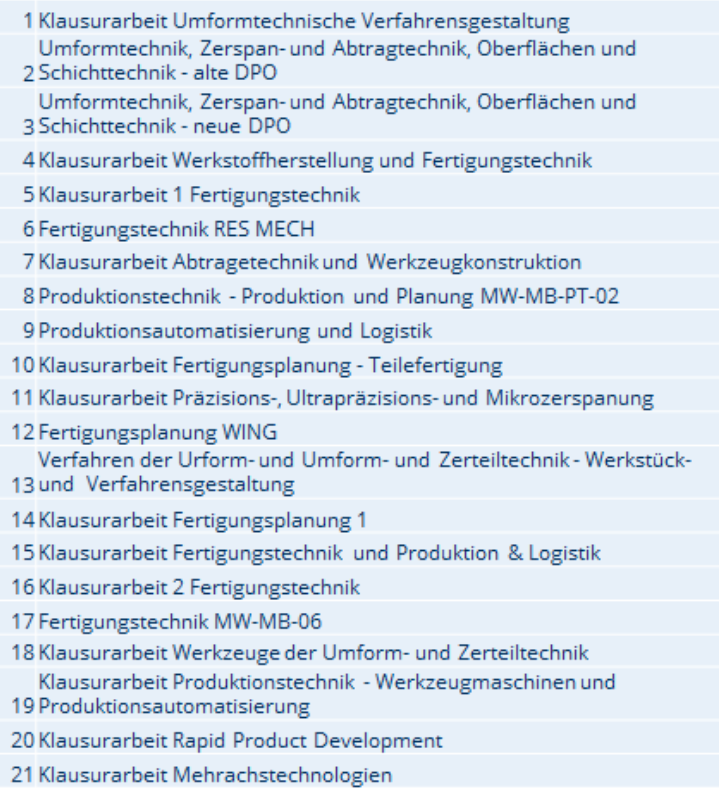

Abb. 1: Online-Klausuren im Wintersemester 2020/21 (jeweils Probe- und Abschlussklausur

Die Ursache für diese hohe Anzahl an Klausuren liegt auch in der Übergangsphase zwischen verschiedenen Diplomprüfungsordnungen (DPO) im Bereich des Maschinenwesens sowie dem Wunsch der Universitäts- und Fakultätsleitung, in jedem Semester den Studierenden alle Prüfungsleistungen zu ermöglichen.
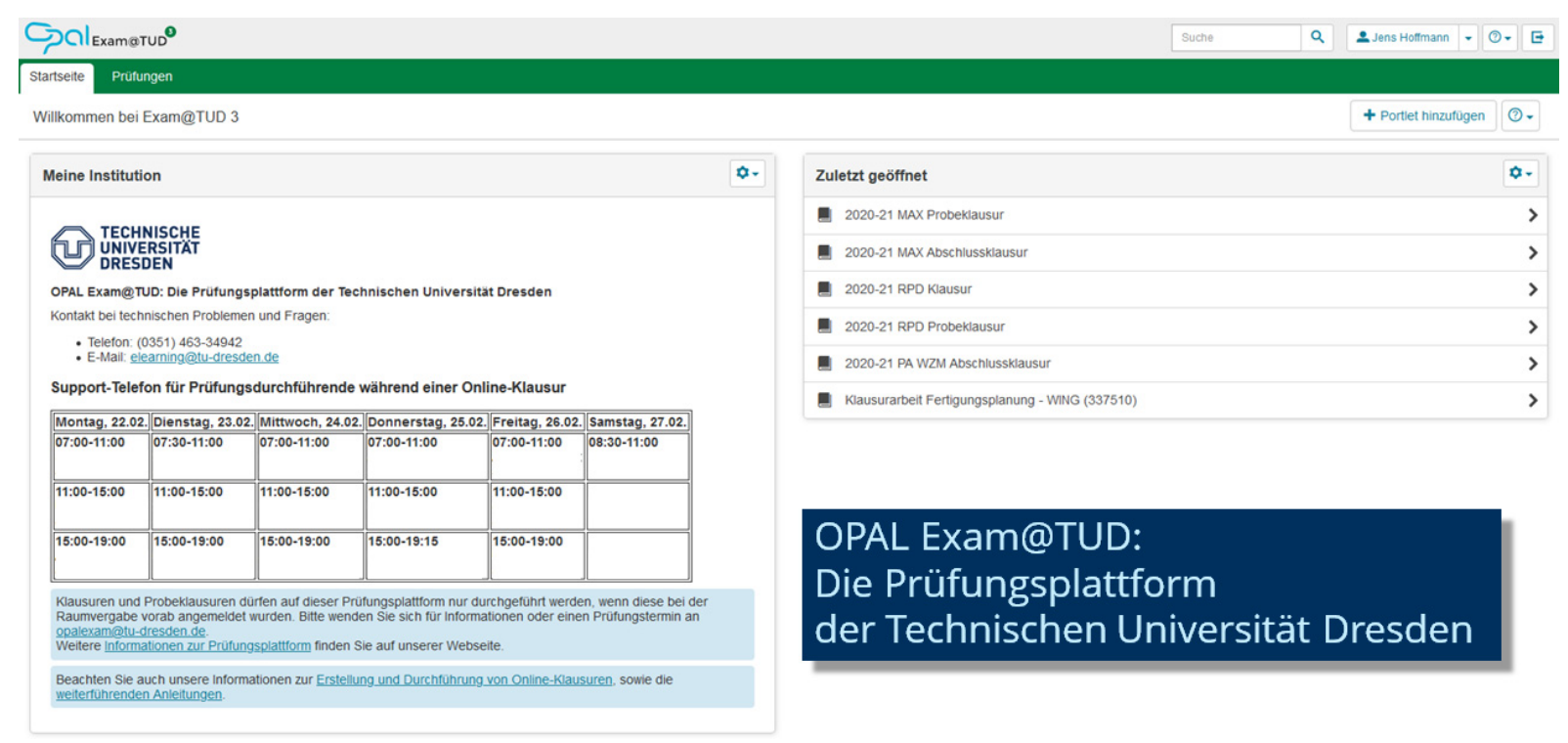

Abb. 2: Startansicht der Prüfungsplattform EXAM3

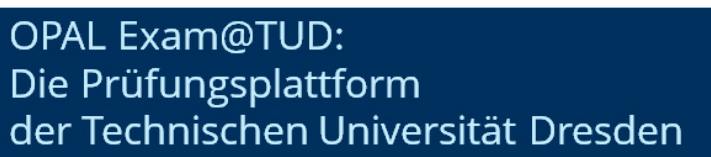

OPAL Exam@TUD:

der Technischen Universität Dresden 


\section{Verwendete Software}

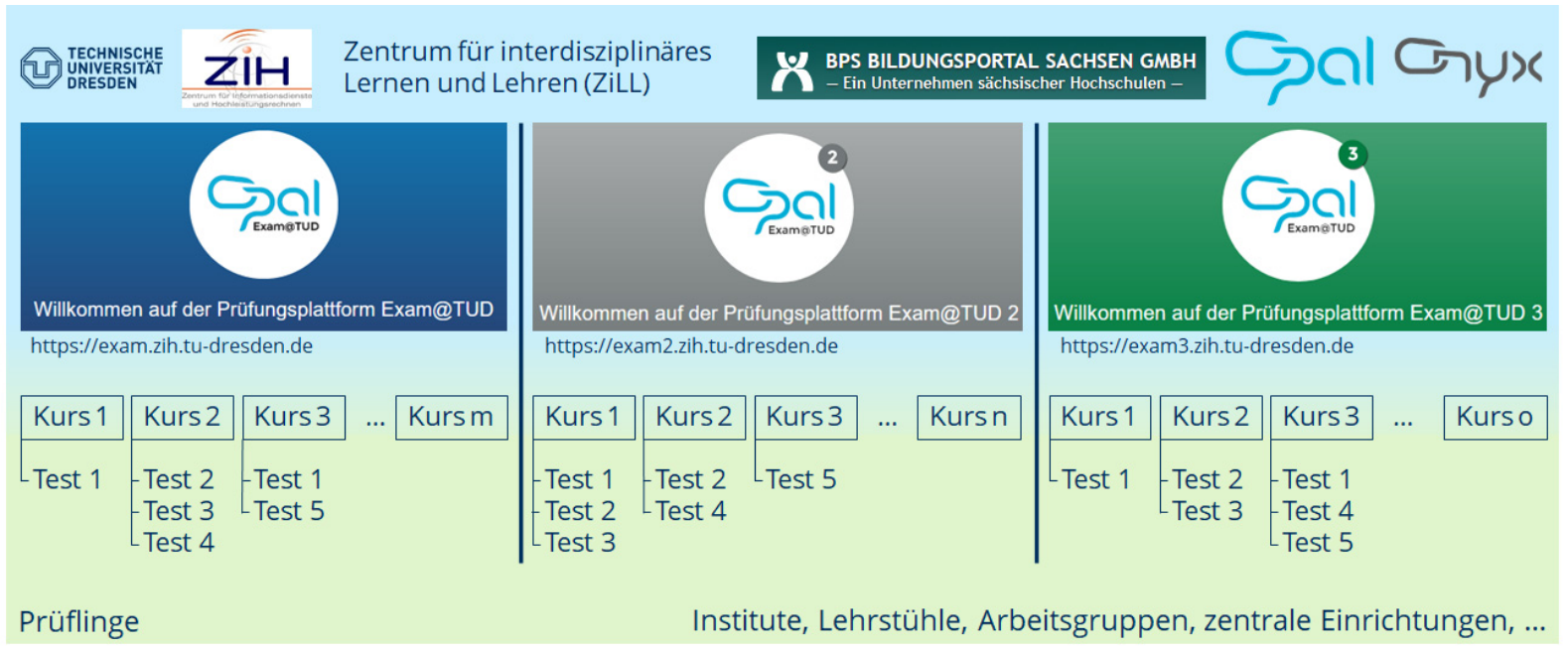

Abb. 3: Struktur der Prüfungsplattform EXAM in der Prüfungsperiode nach dem Wintersemester 2020/21

Wie bereits erwähnt wird an der TU Dresden die Prüfungsplattform EXAM@TUD [1] mit der Software ONYX der BPS GmbH [2] verwendet. Nachdem im Sommer 2020 nur die Plattform EXAM genutzt werden konnte, stehen jetzt zusätzlich EXAM2 und EXAM3 zur Verfügung (Abb. 3). Laut Auskunft des ZILL (Zentrum für interdisziplinäres Lernen und Lehren) [3] können jeweils bis zu 500 Teilnehmer gleichzeitig aktiv sein. Aus den bisherigen Erfahrungen kann diesen Plattformen unter Verantwortung des $\mathrm{ZIH}$ eine hohe Stabilität und Zuverlässigkeit bescheinigt werden.

Allerdings verliert sich durch den Einsatz dreier paralleler Plattformen ohne softwaremäßige Verknüpfung schnell der Überblick für den Prüfenden und der Aufwand für den Datentransfer ist relativ hoch. Aktuell muss ein Kurs von einer Plattform als gepacktes Archiv heruntergeladen und anschließend auf eine der beiden anderen Plattformen hochgeladen werden. Hier sollte nachgebessert werden, um die Plattformen, wie in Windows, üblich als parallele Laufwerke nutzen und auch Verknüpfungen zwischen Elementen (Kurse, Tests) verschiedener Plattformen ermöglichen zu können.

\section{Abgrenzung}

Die Durchführung der Prüfungen erfolgt auf Basis der gültigen Prüfungsordnung des jeweiligen Bereiches. Dabei läuft im Vorfeld das in Abb. 4 dargestellte Prozedere ab.

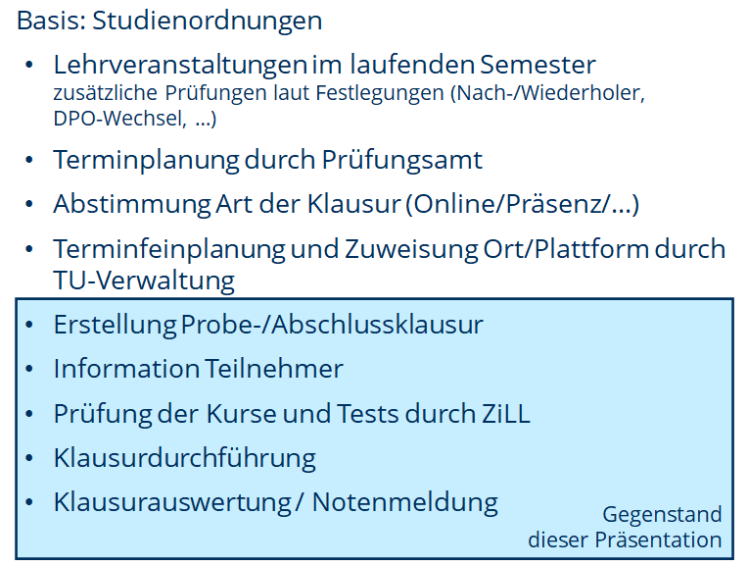

- Lehrveranstaltungen im laufenden Semester zusätzliche Prüfungen laut Festlegungen (Nach-/Wiederholer DPO-Wechsel, ...)

- Terminplanung durch Prüfungsamt

- Abstimmung Art der Klausur (Online/Präsenz/...)

- Terminfeinplanung und Zuweisung Ort/Plattform durch TU-Verwaltung

\begin{tabular}{ll|}
\hline - Erstellung Probe-/Abschlussklausur & \\
- Information Teilnehmer & \\
- Prüfung der Kurse und Tests durch ZiLL & \\
- Klausurdurchführung & \\
- Klausurauswertung/ Notenmeldung renstand \\
\end{tabular}

Abb. 4: Abgrenzung des in diesem Paper betrachteten Bereiches der Prüfungsvorbereitung, -durchführung und -auswertung

Die in der Abbildung entsprechend gekennzeichneten Schritte sollen im Folgenden näher betrachtet werden. Sie weichen von dem bei Präsenzklausuren gewohnten Ablauf deutlich ab. 


\section{Prüfungsvorbereitung}

Digitale Prüfungen erfordern eine andere, meist arbeitsaufwändigere technische und informationstechnische Vorbereitung als bekannte Präsenzklausuren.

Generell besteht eine digitale Prüfung auf ONYX-Basis aus einem OPAL-Kurs und einem oder mehreren Testbausteinen mit jeweils einem ONYX-Test. Ein Test, der die jeweiligen Aufgaben enthält, kann bei Bedarf in verschiedenen Kursen verwendet werden.

Innerhalb der Kurse erfolgen die Steuerung der Prüfung sowie die Verwaltung der Teilnehmer. Dazu sind verschiedene Voreinstellungen und Verknüpfungen einzurichten.

Im ONYX werden die einzelnen Aufgaben erstellt und eingebunden. Diese können nach bestimmten Kriterien in Sektionen zusammengefasst werden.

Die Verwaltung der Teilnehmer und deren Ergebnisse erfolgt im Rahmen des Kurses.

Zusammenfassend kann gesagt werden, dass der Vorbereitungsaufwand für eine digitale Prüfung meist wesentlich höher als für eine Präsenzklausur ist.

\section{Kurs}

Basis für die Erstellung einer Online-Klausur ist ein OPAL-Kurs (Abb. 5). Dieser kann neu oder als Kopie angelegt bzw. wiederverwendet werden. Bei einer Wiederverwendung sind die Nutzerdaten zu aktualisieren. Alte Daten gehen dabei verloren. Aus Sicht der Aufbewahrung der Ergebnisse früherer Online-Klausuren entsprechend den geltenden Vorschriften ist es empfehlenswert, mit einer neu erstellten Kopie des Kurses zu arbeiten. Bei Probeklausuren kann durchaus davon abgewichen werden, da hier der Aspekt der Aufbewahrung der Nutzerdaten keine Rolle spielt.

In Verbindung damit steht ein wesentliches Problem bei der Nutzung von ONYX wie bei OPAL insgesamt: Die Dateiverwaltung (Abb. 6). Es ist nicht möglich, als Nutzer auf Kursebene mit Ordnern oder Verzeichnissen zu arbeiten und damit den vorhandenen Datenbestand sinnvoll zu strukturieren. Bisher kann nur über den Namen des Kurses eine sinnvolle Strukturierung erfolgen (z.B. Voransetzung eines Präfixes des jeweils relevanten Prüfungszeitraumes bei der Kursbezeichnung). Bei ca. 60 Kursen wird es daher schnell unübersichtlich. Hier besteht dringender Handlungsbedarf seitens der BPS GmbH.

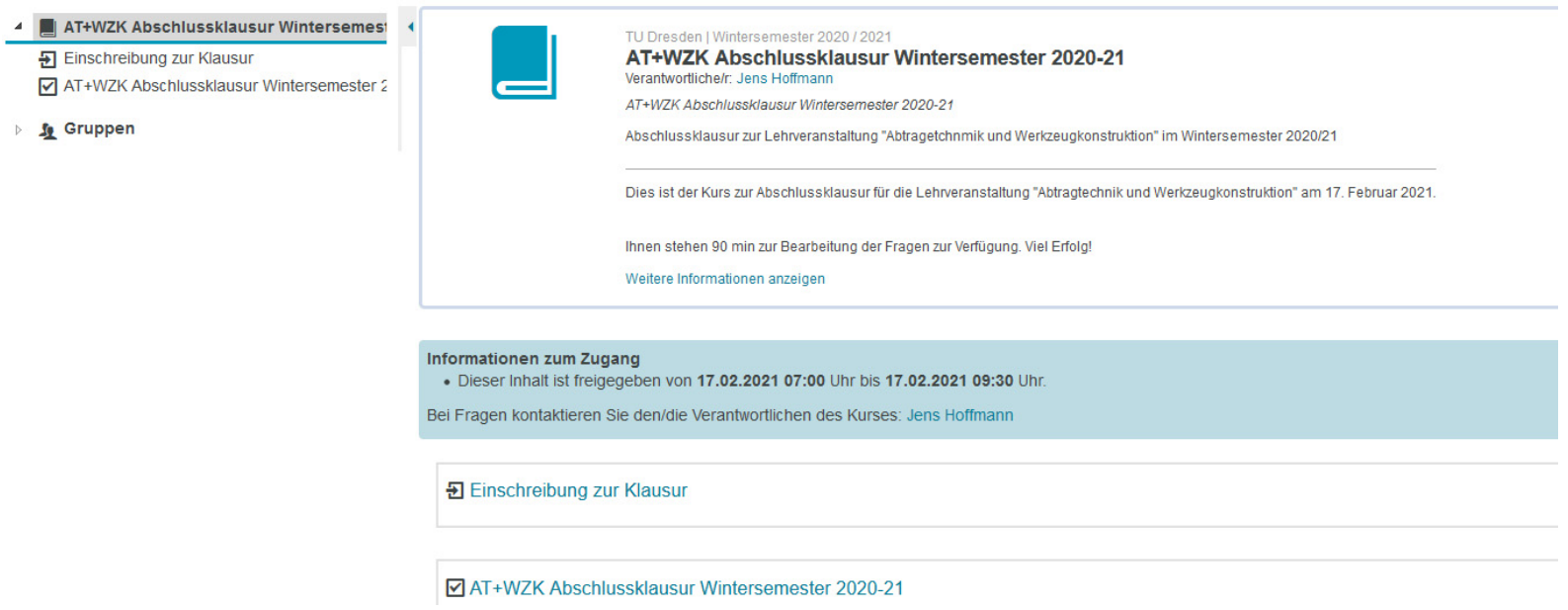

Abb. 5: Startansicht eines Online-Prüfungskurses 


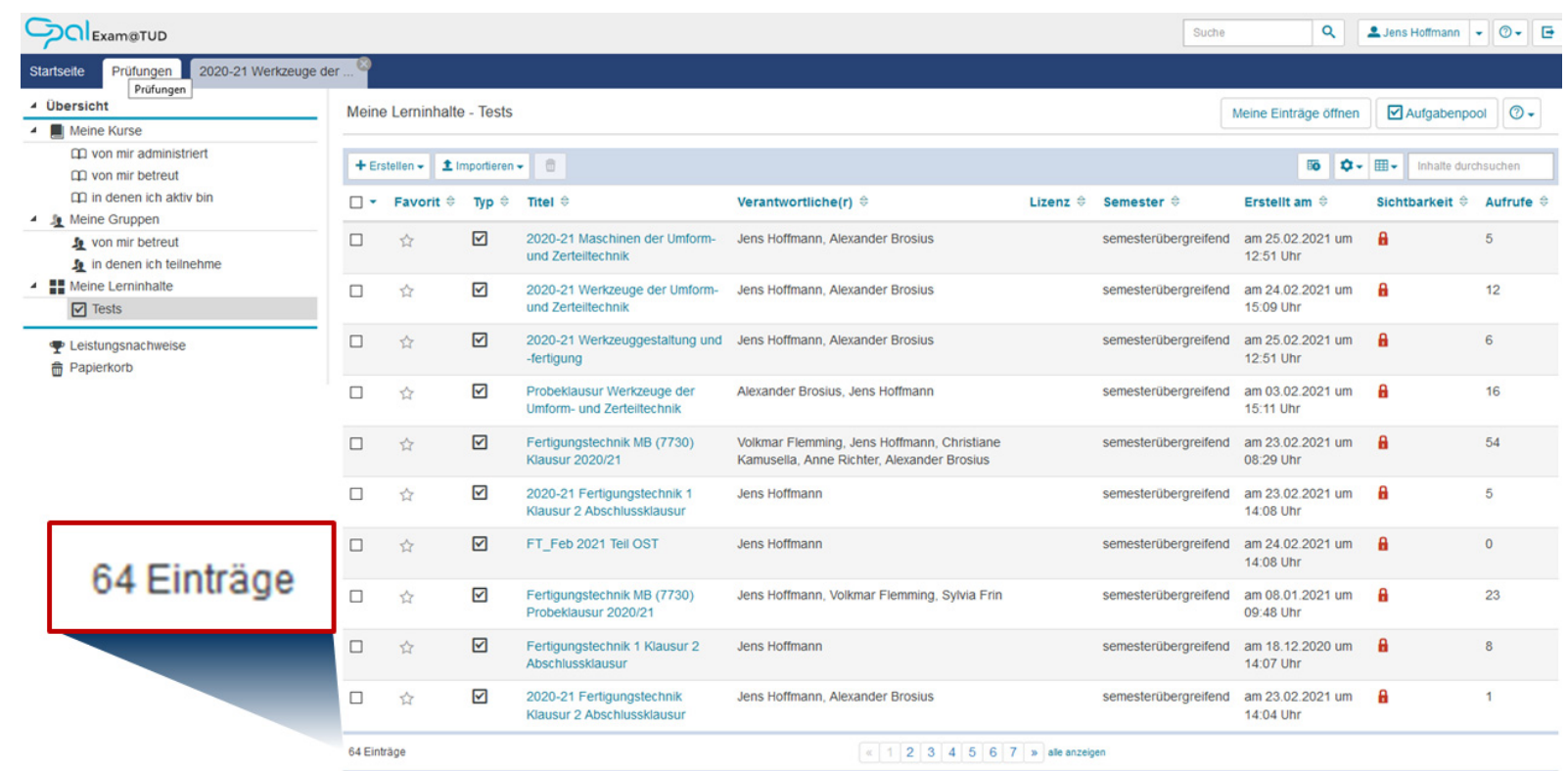

Abb. 6: "Kursverwaltung" im EXAM/ONYX

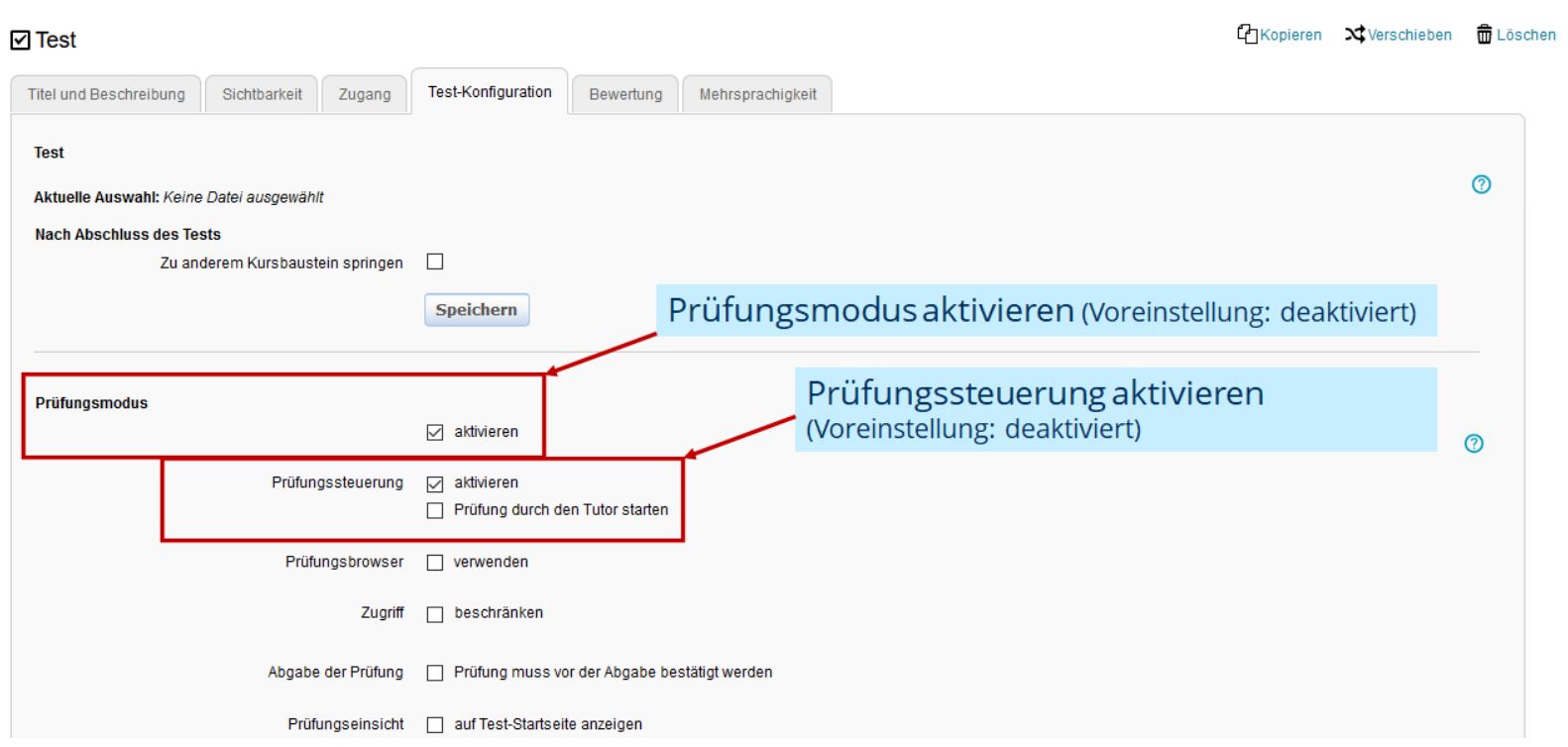

Abb. 7: Einstellungen bei der Test-Konfiguration (Beispiel)

Für unsere Klausuren verwenden wir, wie bereits erwähnt, neue oder kopierte Kurse. Diese müssen allerdings jedes Mal eingerichtet werden, da leider die Voreinstellungen des Systems EXAM/ONYX nicht den Anforderungen der TU Dresden bzw. des ZILL entsprechen (Abb. 7). Speziell für die Sichtbarkeit der Auswertung der durchgeführten Tests ist eine Anpassung erforderlich. Auch hier besteht Nachbesserungsbedarf bei der Weiterentwicklung der Software für die Zukunft.
Teilnehmer der Klausuren können entsprechend den Vorgaben des Systems eingeladen werden oder sich selbständig einschreiben. Am Lehrstuhl wird im Allgemeinen letztere Variante in Verbindung mit einer Bestätigung der Teilnahmebedingungen zur Prüfung bevorzugt. Es ist zu beachten, dass sich die Teilnehmer nicht selbständig wieder austragen können (leider keine Voreinstellung im System, muss manuell eingerichtet werden, Abb. 8). 


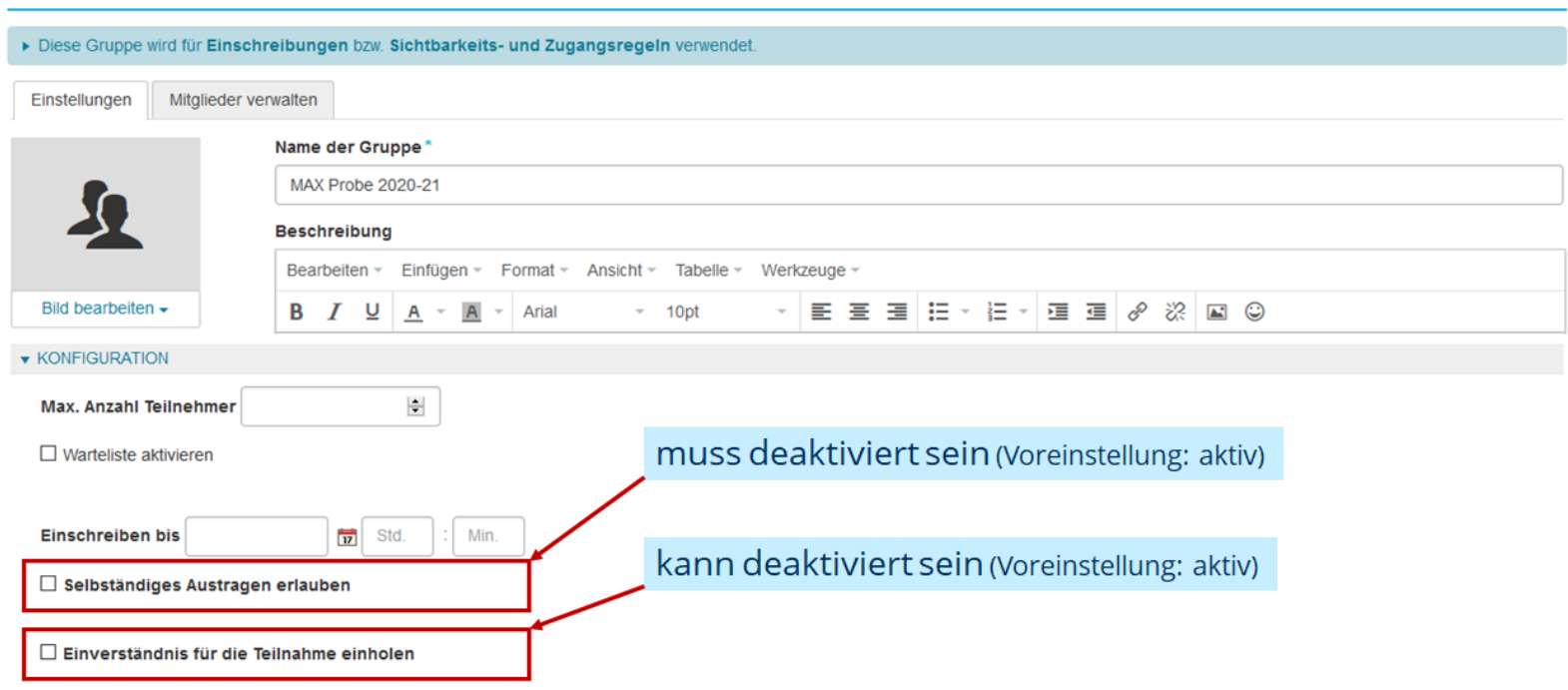

Abb. 8: Einstellungen bei der Mitgliederverwaltung

Zu beachten ist, dass bestimmte Einstellungen zur Prüfungssteuerung im OPAL-Kurs (z.B. Anzahl der Lösungsversuche im Testbaustein) oder im ONYX-Test (z.B. Prüfungsdauer) zu realisieren sind. Bei der Anzahl der Lösungsversuche ist auch eine Festlegung an beiden Stellen möglich, die Einstellung im ONYX-Test hat jedoch keinen Einfluss auf den Klausurablauf. An dieser Stelle ist eine Synchronisierung der Einstellungen zwischen OPAL und ONYX unbedingt erforderlich.

\section{Test}

Der ONYX-Test beinhaltet die verschiedenen Aufgaben einer Klausur. Wie bereits erwähnt können für eine Klausur ein oder mehrere Tests verwendet werden. Jeder einzelne ONYXTest muss durch einen Testbaustein eingebunden werden. Die Nutzung eines ONYX-Tests in verschiedenen Testbausteinen oder OPALKursen ist möglich und kann den Arbeitsaufwand reduzieren.

Innerhalb eines ONYX-Tests können Aufgaben zu Sektionen zusammengefasst werden (Abb. 9). Hier ist eine getrennte Ermittlung der erreichten Punkte möglich. Diese werden leider bisher nur in der Prüfungsauswertung angezeigt, können aber nicht für die Weiterverarbeitung, z. B. als Excel-Datei, gespeichert wer- den. Es bleibt nur der manuelle Export der Daten - das Fehlen dieser Funktionalität ist für den Nutzer nicht nachvollziehbar.
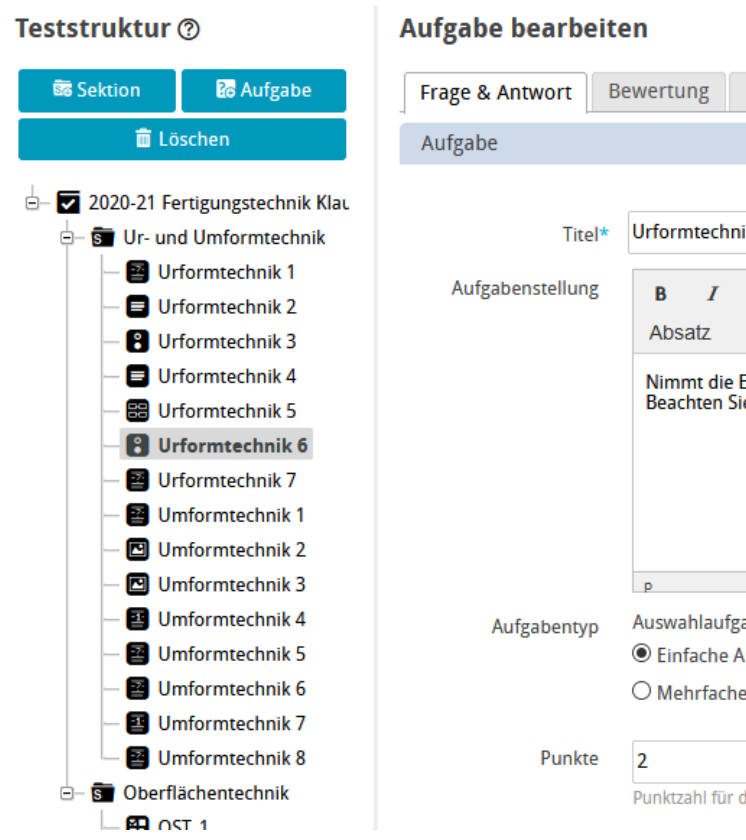

Abb. 9: Aufbau eines Tests mit Sektionen

Im Test können, wie bereits erwähnt, verschiedene Festlegungen zur Prüfungssteuerung getroffen werden. Dazu zählen z.B. die Auswahl der Aufgaben, deren Reihenfolge sowie die Art und Weise der Bearbeitung durch den Prüfling (linear/frei). 
Diese Definitionen erfordern fundierte Kenntnisse über die Wirkung der und Inhalte der Parameter, Erfahrung und Konzentration bei der Einstellung. Wünschenswert wäre eine bessere Voreinstellung der Parameter speziell nach den Vorgaben der TU sowie eine bessere Beschreibung der Funktionalitäten.

\section{Aufgabentypen und zugehörige Schwierigkeiten}

Es stehen verschiedene Aufgabentypen zur Nutzung bereit (Abb. 10). Diese unterscheiden sich naturgemäß von denen, die aus Präsenzprüfungen bekannt sind. Ausnahme ist der sogenannte Freitext. Die weiteren Aufgabentypen sind gut nutzbar. Eine entsprechende Erläuterung ist im ONYX-Editor hinterlegt sowie über das ZILL verfügbar. Es gibt dazu an unserm Lehrstuhl noch einige spezielle Erfahrungen, auf die jetzt eingegangen werden soll.

\section{Neue Aufgabe erstellen}

\begin{tabular}{l|l|}
\hline \multirow{4}{*}{ Titel* } & Neue Aufgabe \\
\hline Aufgabentyp** & Basis \\
\hline & Auswahlaufgabe \\
\hline & Lückentextaufgabe \\
Freitextaufgabe \\
Erweitert \\
Einfache Zuordnung (Drag-and-Drop) \\
Mehrfache Zuordnung (Matrix) \\
Grafische Zuordnung \\
Reihenfolgeaufgabe \\
Hotspotaufgabe \\
Fehlertextaufgabe \\
Textboxaufgabe \\
Uploadaufgabe \\
Spezifisch \\
Numerische Eingabe (Lückentext) \\
Berechnung (Lückentext) \\
Formelvergleich (Lückentext) \\
Regulärer Ausdruck (Lückentext) \\
\hline
\end{tabular}

Abb. 10: Verfügbare Aufgabentypen

Fragen können zwischen verschiedenen Tests kopiert oder verschoben werden. Dies kann über die Funktion „extrahieren“ und anschließenden Import erfolgen. Soll dies noch zwischen verschiedenen EXAM@TUD-Plattformen erfolgen ist zusätzlich noch, wie bereits bei den Kursen beschrieben, ein Down- und ein Upload erforderlich. Dies geht sicherlich auch einfacher und zeitgemäßer und ist damit ein weiterer Wunsch für zukünftige Entwicklungen.

\subsection{Problematik Auswahlaufgabe Mul- tiple Choice}

Bei Auswahlaufgaben stehen die Varianten Single und Multiple Choice zur Verfügung. Während bei einer richtigen Lösung (Single Choice) keine wesentlichen Probleme bei der Erstellung auftreten, verhält es sich bei Multiple Choice anders. Hier sind die Vorgaben der MC-Richtlinie der TU Dresden zu beachten [4]. Dies bedingt bei der Bewertung eine Reihe von Einstellungen, die zunächst nicht unbedingt logisch erscheinen. Die Frage ist, warum diese hochschulspezifischen Anforderungen nicht durch Voreinstellungen des ONYX-Systems besser abgefangen werden. Auch hier besteht aus unserer Sicht Nachbesserungsbedarf.

\subsection{Problematik Lückentext}

Eine weitere mögliche Aufgabenform ist der Lückentext. Dabei wird in einem Text ein Wort oder eine Wortgruppe bzw. eine Zahl durch eine Lücke ersetzt. In diese ist durch die Prüfungsteilnehmer die entsprechende Lösung einzutragen. Diese Eingabe wird vom System mit einer Vorgabe und eventuell definierten Alternativen verglichen. Trotz der Möglichkeit zur Programmierung einer "Unschärfe“ (Buchstabenabweichung, Groß-/Kleinschreibung) ist es schwierig, alle möglichen richtigen Antwortoptionen zu erfassen. Dies führt zu häufig notwendigen manuellen Nachkorrekturen und erhöht damit den Aufwand. Sinnvoll ist die Lücke bei exakten Werten (z.B. Zahlen), sonst eher misslich.

\subsection{Problematik Freitext}

Der bekannten Fragestellung bei Präsenzklausuren sehr ähnlich kommt der Aufgabentyp "Freitext". Hier können zu einer Frage durch den Teilnehmer in ein Textfeld entsprechende Ausführungen eingegeben werden. Diese müssen nach der Klausurdurchführung durch den Prüfer manuell bewertet werden. Eine Automatisierung der Bewertung ist bei diesem Aufgabentyp nahezu unmöglich.

Der Prüfer sollte bei der Erstellung einer Aufgabe dieses Typs verhindern, dass der Teilnehmer mittels Copy\&Paste einfach Texte aus anderen Anwendungen (pdf-Dateien, Internet, ...) kopieren kann, was nicht erlaubt ist und einen 
Betrugsversuch darstellt. Dies kann durch Setzen einer entsprechenden Option erfolgen. Diese ist jedoch auch eine wenig versteckt („Einfügen externer Inhalte zulassen“, Abb. 11).

Aufgabe bearbeiten
\begin{tabular}{|l|l|l|}
\hline Frage \& Antwort & Bewertung & Feedback Optionen \\
\hline Optionen
\end{tabular}

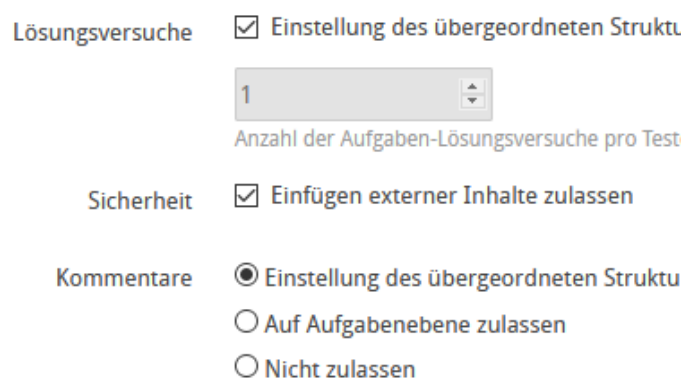

Abb. 11: Anzeige der Sektionsergebnisse

Im Unterschied zu den anderen Aufgabentypen ist bei Freitext-Aufgaben der Vorbereitungsaufwand sehr gering, dafür muss mehr Zeit in die Auswertung gesteckt. Speziell bei Teilnehmerzahlen von größer 20 ... 50 ist dieser Aufwand individuell zu hinterfragen.

\subsection{Hochladen von Dateien}

Neben Freitext-Aufgaben besteht aktuell auch die Möglichkeit, Dateien im Rahmen einer Klausur hochzuladen. Damit können zum Beispiel Lösungswege oder Skizzen von den Prüflingen erfasst und zur Bewertung bereitgestellt werden. Somit besteht die Möglichkeit, den Bearbeitungsweg besser nachzuvollziehen und Folgefehler zu berücksichtigen.

Abhängig von der Dateigröße und der verfügbaren Internetverbindung kann dieses Mittel jedoch problematisch werden. Der Bewertungsaufwand ist ähnlich hoch wie bei Freitextaufgaben.

\section{Vorbereitung zur Durchführung}

Zunächst sollte vor dem Prüfungstermin nicht vergessen werden, die erstellten OPAL-Kurse (nicht ONYX-Tests) öffentlich zu machen (Einstellung im EXAM@TUD) sowie die Sichtbarkeits- und Zugangsregeln für den Kurs korrekt zu setzen. Speziell die Zeit und die Zugehörigkeit zu bestimmten Teilnehmergruppen ist hierbei zu beachten.

Nach der Erstellung der Kurse und Tests ergibt sich die Problematik der Informationsweitergabe an potenzielle Teilnehmer über Modalitäten und den Link zum Kurs. Eine aktive Unterstützung durch Prüfungsamt und Studienbüro bei Onlineklausuren bezüglich der Information der Teilnehmer erfolgt leider nicht.

Fertig vorbereitete Klausuren werden dankenswerter Weise durch die Mitarbeiter:innen des ZILL vor dem Prüfungstermin noch einmal geprüft. Erforderliche Korrekturen sollten noch vor der Klausur durchgeführt werden. Dass manchmal dafür nicht besonders viel Zeit zur Verfügung steht, müssen sich die Kursverantwortlichen allgemein selbstkritisch ankreiden lassen, da die Fertigstellung der benötigten Kurse und Tests doch teilweise sehr "zeitnah" erfolgt und damit kaum Zeit zur Überprüfung durch das ZiLL bleibt.

\section{Teilnehmerverwaltung}

Ein bisher noch nicht näher betrachteter Punkt bei der Durchführung der Online-Klausuren ist die sich ändernde Aktualität der Teilnehmer. Zunächst sind die bereits erwähnten Einstellungen in der Nutzerverwaltung sicherzustellen.

Für die Erfassung der aktiven Teilnehmer gibt es zwei Möglichkeiten.

a) Wenn im Vorfeld die kompletten aktuellen Teilnehmerlisten über Hisqis oder Selma (Verwaltungssysteme für studentische Leistungen an der TU Dresden) bereitstehen können, diese für eine Einladung und die anschließende Klausurteilnahme genutzt werden. Dabei sind diese Daten in der Teilnehmerverwaltung zu erfassen und nur diesen Teilnehmern ist der Zugang zu ermöglichen. Leider ist dieser Weg durch die Vielfalt der Teilnehmerkreise auch aus anderen Bereichen und die damit verbundene Unvollständigkeit und Unübersichtlichkeit der Daten nicht immer gegeben.

b) Alternativ kann eine automatische Einschreibung beim Zugang zum Kurs definiert werden. Damit kann jeder, der die Zugangsdaten besitzt (Link und Passwort) den Test absolvieren. Eine Kopplung der 
automatischen Einschreibung mit einer Zustimmung zu der aktuellen Prüfungsbedingungen ist möglich (Baustein „Einschreibung zur Klausur"). Es besteht dabei aber die Gefahr, das auch Unberechtigte Zugang erhalten.

Im Nachgang zur Prüfung müssen dann die Ergebnisse der Teilnehmer teilweise sehr arbeitsaufwändig den verschiedenen Hisqis- und Selma-Datenbeständen zugeordnet werden.

\section{Durchführung der Online-Klausur}

Die Durchführung der Online-Klausuren hat sich im Wintersemester 2020/21 als weitgehend problemlos erwiesen. Wenn nicht menschliche Fehler (falscher Test eingebunden, falsche Einstellungen, ...) zu Problemen führen, sind die drei Plattformen stabil durchgelaufen. Der Verantwortliche hat wenige Eingriffsmöglichkeiten (Test neustarten, beenden, Zeitzugabe), bei Störungen der Internetverbindung jedoch gar keine. Auch ist die Überwachung der Arbeitsweise der Teilnehmer (Täuschungsversuche) nahezu unmöglich.

In seltenen Fällen musste über das Bewertungstool die Anzahl der Prüfungsversuche (die normalerweise eins nicht überschreiten sollte) manuell bei Abbruch der Bearbeitung der Klausur zurückgesetzt werden.

Unklar ist aktuell die Zeitsteuerung der Klausur. Es gibt einen zeitlichen definierten Zugang zum OPAL-Kurs sowie eine Begrenzung der Bearbeitungsdauer des ONYX-Tests. Bedeutet der Zugangszeitraum nur die Begrenzung der Startzeit der Klausur durch den Studierenden oder begrenzt dieser auch die Bearbeitungsdauer auf das Gesamtzeitfenster? Wie ist dies mit dem zugewiesenen Zeitfenster für die Online-Klausur abzustimmen? Hierzu bedarf es der Klärung seitens des Prüfungsausschusses.

\section{Auswertung der Online-Klausuren}

Nach der Durchführung der Online-Klausuren ist die Auswertung erforderlich. Auch dazu sind verschiedene Hilfsmittel verfügbar. Zunächst sollten die manuellen Bewertungen (Freitext, Lückentext, Datei-Upload) durchgeführt werden. Diese werden im System nach manueller Eingabe gemeinsam mit allen anderen verwaltet. Anschließend stehen die Ergebnisdaten zur Auswertung im System bereit. Bei einem durchgehenden Test (z.B. ohne Nutzung von Sektionen für getrennte Teil-Tests) kann der Ergebnisexport über den Download im Bewertungswerkzeug erfolgen. Wenn verschiedene Teilgebiete getrennt bewertet werden sollen und dabei die Sektionen in einem Test genutzt wurden, muss über die Dateiarchivierung agiert werden. Dabei entsteht eine sehr umfangreiche Datei mit sämtlichen Informationen. Allerdings ist diese aktuell nur im csv-Format verfügbar und muss erst nach Excel transformiert werden. Am Bildschirm werden die Teilergebnisse angezeigt (Abb. 12) aber leider nicht gespeichert. Auch hier wäre eine Verbesserung der Nutzerfreundlichkeit wünschenswert.

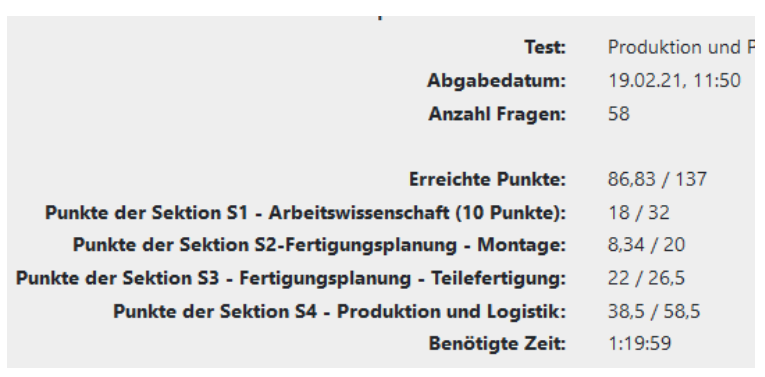

Abb. 12: Anzeige der Sektionsergebnisse

\section{Konflikt DPO - ONYX}

Neben anderen wesentlichen Festlegungen erfolgt durch die DPO die Zuordnung der einzelnen Lehrveranstaltungen zu Modulen. Für diese werden die Kriterien für die entsprechenden Prüfungsleistungen definiert. Zu einem Modul können eine oder mehrere Lehrveranstaltungen gehören.

Ein Bespiel ist die Fertigungstechnik im 1. Semester (Abb. 13). 
- Beispiel: Fertigungstechnik1. Semester MW-MB-06, knapp 400 Teilnehmer

- 4 Haupt-Teilgebiete: Ur- und Umformtechnik, Zerspan- und Abtragtechnik, Fügetechnik, Oberflächen- und Schichttechnik, "Sonstige“

3 ... 4 Professuren fachlich beteiligt $\rightarrow$ getrennte Aufgaben und Auswertung

- Prüfungsdauer laut DPO: 180 min (keine Aufteilung auf Teilgebiete genannt)

\section{- Umsetzung mit EXAM}

- ein Testbaustein pro Teilgebiet

- Aufgabenstellung und Auswertung unkritisch (je Testbaustein ein Test)

- Zeitbegrenzung kritisch (Zeit je Test bedeutet Aufteilung der $180 \mathrm{~min}$ )

- ein Testbaustein für alle, Arbeit mit Sektionen im Test

- Aufgabenstellung unkritisch

- Auswertung äußerst aufwändig

(keine Ausgabe der Ergebnisse je Sektion, Arbeit mit Excel-Datei aus Datenarchivierung „schwierig”)

- Zeitbegrenzung unkritisch (Gesamtzeit 180 min für Gesamttest)

Abb. 13: Problematik verschiedene Teilgebiete in einem Test

Im Modul MW-MB-PT-03 sind die beiden Lehrveranstaltungen Werkzeugmaschinen und Produktionsautomatisierung enthalten, deren zeitlicher Umfang identisch ist. Die Gesamtprüfungsdauer beträgt $180 \mathrm{~min}$. Die beiden Teile werden von verschiedenen Professuren durchgeführt. Sinnvoll ist es, zwei inhaltlich getrennte Teilprüfungen durchzuführen und anschließend die Teilnoten zu verrechnen. Wie ist eine Umsetzung in EXAM@TUD möglich?

Variante 1 wäre die Nutzung zweier getrennter Tests und entsprechend von zwei Testbausteinen. Es werden dann die Prüfungsergebnisse auch getrennt ermittelt. Eine übergreifende Prüfungszeit kann aber nicht definiert werden, sondern nur die Zeiten je Teilgebiet, was nach DPO nicht rechtens ist.

Variante 2 wäre ein Test mit zwei entsprechenden Sektionen. Es wird eine Gesamtprüfungszeit definiert und der Prüfling teilt sich diese selbst ein. Problematisch dabei ist, dass für die beiden Sektionen keine getrennten Ergebnisse ermittelt werden können. Es kann nur eine sehr große unübersichtliche Datei zur manuellen Auswertung über die Dateiarchivierung erzeugt werden. Wie bereits erwähnt, werden Sektionsergebnisse zwar angezeigt, aber nicht gespeichert.

\section{Problemfälle Hisqis / Selma}

Ein wesentliches Problem sind die fehlenden Schnittstellen zu den im Prüfungsamt bzw. Studienbüro verwendeten Softwareplattformen
Hisqis und Selma. Alle Systeme arbeiten mit der Ein- und Ausgabe von Daten im Excel-Format, damit enden aber auch leider schon die Gemeinsamkeiten. Struktur und Inhalt unterscheiden sich wesentlich, womit ein manueller Aufwand zur entsprechenden Datentransformation in mehr oder weniger großem Umfang anfällt.

\section{Zusammenfassung}

Insgesamt ist im Ergebnis der aktuellen Prüfungsperiode festzustellen, dass die OnlineKlausuren sicher und stabil gelaufen sind. Es muss mehr Zeit für die Vorbereitung der Klausuren gegenüber Präsenzprüfungen aufgewendet werden, auch sind die Fragstellungen den Möglichkeiten der Software anzupassen. Ob dies für die Studierenden positiv oder negativ wirkt, ist fach- und sachabhängig.

Der Aufwand für die Auswertung der Klausuren kann zwischen sehr gering und umfangreich schwanken. Die Ursachen dafür wurden in den vorherigen Abschnitten ausführlich dargelegt. Offen bleibt die Frage nach der rechtssicheren Feststellung von Betrugsversuchen. Diese können durch geeignete Maßnahmen bei der Gestaltung der Klausuren eingeschränkt, aber nicht vollständig ausgeschlossen werden. Es muss davon ausgegangen werden, dass den Teilnehmern alle Unterlagen (Skripte, Bücher, Internet, ...) während der Klausur zur Verfügung stehen. Dies ist bei der Gestaltung der Aufgaben und der Prüfungssteuerung zu bedenken. 
Für die genutzte Plattform EXAM@TUD hier noch einmal zusammenfassend die aus unserer Sicht vorrangigen Änderungs- bzw. Entwicklungswünsche:

\section{Nutzersysteme EXAM / EXAM2 / EXAM3}

- Nutzung der Kurse / Test über Plattformgrenzen hinweg bzw. Verwendung einer einheitlichen Plattform

- Transfer der Kurse / Tests zwischen den Plattformen vereinfachen (nicht mehr über Download/Upload mit lokalem Speicher)

- Aufbau einer Ordnerstruktur für Kurse zur Erhöhung der Übersichtlichkeit (analog zu Aufgabenpool)

\section{OPAL-Kurse / ONYX-Tests}

- Voreinstellungen anpassen

- Nutzerverwaltung mit-Sperrung desselbständigen Austragen und dem Deaktivieren des Sendens einer Information über Einschreibung

- Prüfungsversuche limitieren

- Anzeige Auswertung der Prüfung deaktivieren

- Prüfungssteuerung aktivieren

- Einstellungen abstimmen zwischen Kurs und Test (Zeit für Zugriff, Anzahl der Zugriffe, ...)

- Ende der Zugriffszeit bedeutet Ende der Bearbeitungszeit des Tests

- MC-Aufgaben: Voreinstellungen an MCRichtlinie der TU Dresden anpassen

- Direktausgabe der Sektionsergebnisse in Datei ermöglichen

- Bewertungstool an Realität anpassen / flexibler machen

- Ausgabe von Aufgaben-/Teil-/Sektionsergebnissen in Datei ermöglichen

- Abstimmung / Umsetzung Studienordnungen mit den entsprechenden Softwaremöglichkeiten

- Transfer von Aufgaben zwischen Tests vereinfachen

\section{Prüfungsorganisation, -verwaltung}

- Informationen für Studenten auch über Prüfungsamt (Link, Termin)
- Bereitstellung Kontaktdaten aus Hisqis / Selma

- einheitliche Datenformate oder Direktschnittstellen Hisqis - Selma - ONYX

Diese Wünsche sollten durch die Fakultät gesammelt und über die TU oder direkt an die BPS GmbH übermittelt werden.

Ein Dank gilt abschließend den Kolleg:innen des Prüfungsamtes Maschinenwesen für die flexible Unterstützung bei der Klärung verschiedenster Probleme der Organisation und Verwaltung der Prüfungen, den Mitarbeiter:innen des ZILL für Ihre aktive Unterstützung bei der Vorbereitung und Durchführung der Online-Klausuren durch Schulungen, Unterlagen und kritischen Blick auf die Kurse sowie auch dem ZIH für die stabile Rechentechnik.

\section{Literatur}

[1] https://exam.zih.tu-dresden.de, https://exam2.zih.tu-dresden.de, https://exam3.zih.tu-dresden.de

[2] https://www.bps-system.de/

[3] https://tu-dresden.de/tu-dresden/organisation/rek torat/prorektor-bildung/ZiLL

[4] https://tu-dresden.de/tu-dresden/organisation/rek torat/prorektor-bildung/zill/e-learning/corona/digi tale-pruefungen/bewertung-von-mc-aufgaben

[5] https://tu-dresden.de/ing/maschinenwesen/res sourcen/dateien/studium/pruefungsamt/folder2015-05-269353339805/mbpt 19 2601.pdf?lang=de; Abruf: 26.02.2021, 07:00 Uhr 